\title{
Galactic planetary nebulae and their central stars
}

\section{Proper motions ${ }^{\star}$}

\author{
F. Kerber ${ }^{1}$, R. P. Mignani ${ }^{2}$, R. L. Smart ${ }^{3}$, and A. Wicenec ${ }^{1}$ \\ ${ }^{1}$ ESO, Karl-Schwarzschild-Str. 2, 85748 Garching, Germany \\ e-mail: [fkerber; awicenec] @eso.org \\ 2 Mullard Space Science Laboratory, University College London, Holmbury St. Mary, Dorking - Surrey, RH5 6NT, UK \\ e-mail: rm2@mssl.ucl.ac.uk \\ 3 INAF, Osservatorio Astronomico di Torino, via Osservatorio 20, 10025 Pino Torinese, Italy \\ e-mail: smart@oato.inaf.it
}

Received 2 July 2007 / Accepted 29 November 2007

ABSTRACT

\begin{abstract}
Context. More than 1500 Galactic planetary nebulae (PNe) are known but only a tiny fraction of them have measured proper motions. To date, the largest set of proper motion (PM) data for PNe is the one by Cudworth (1974), which includes 62 objects 25 of which have PM with significance better than $3 \sigma$ in at least one component.

Aims. With our new compilation of 234 PNe we enlarge - compared to Cudworth's 25 - by almost an order of magnitude the number of PNe and central stars (CSs) with reliably measured proper motion (i.e. with significance better than $3 \sigma$ in at least one component) and confirm some previous measurements.

Methods. We have used all-sky astrometric catalogues available via the Vizier database to collect proper motion information for a sample of objects selected from the catalogue of PNe positions of Kerber et al. (2003a, A\&A, 408, 1029).

Results. We have derived proper motion information for a total of $234 \mathrm{PNe}$ ( 274 when including 40 doubtful candidates). We include all PNe for which a confidence level of at least $3 \sigma$ was achieved in at least one proper motion component. For many objects PM data are available from more than one catalogue and agreement between different catalogues is usually very reasonable providing independent confirmation of the results. For comparison with Cudworth's results we use the proper motion modulus as a metric. We cross-correlated his 62 objects with our master catalogue and found 12 matching objects out of the 18 with $>3 \sigma$ in his list. For these we find good agreement for 10 out of the 12 objects, while we find significant PM for another five objects with PM $\sigma \leq 3$ reported by Cudworth. The number of objects we have in common was limited by several factors discussed in the paper.

Conclusions. With 234 (274 including doubtful candidates) entries our work is the largest available compilation of proper motion data for PNe and their CSs. This compilation opens new opportunities for studies of orbital kinematics of PNe in the Galaxy.
\end{abstract}

Key words. planetary nebulae: general - Galaxy: kinematics and dynamics - catalogs - astrometry

\section{Introduction}

Planetary Nebulae (PN) are the final stage of stellar evolution for the bulk of the Galactic stellar population. While some aspects of the objects have been studied in great detail, others remain largely unknown. A case in point is their proper motion (PM): the most comprehensive study of the proper motion of Galactic PNe was published by Cudworth (1974), who reported new proper motion measurements for 51 bright nebulae based on positions recorded on photographic plates taken about nine years apart (plus another 11 from literature data). The only other proper motion catalogue of PNe published afterwards is the one of Acker et al. (1998), based on Hipparcos data (Perryman et al. 1997) which provides PM data for 19 PNe; only 11 of which have significant ( $3 \sigma$ in at least one component) PM. There are two main difficulties in obtaining good PM measurements for PNe: first, they all are at significant distances from Earth; the closest known PN is Sh 2-216 (Harris et al. 2007) at a distance of about $130 \mathrm{pc}$. Even for this object the PM is rather modest

* Table 3 is only available in electronic form at the CDS via anonymous ftp to cdsarc.u-strasbg.fr (130.79.128.5) or via http://cdsweb.u-strasbg.fr/cgi-bin/qcat?]/A+A/479/155
(Kerber et al. 2004), see also Table 3. Second, the presence of the bright nebulosity around the - usually faint - CS is a complication for measuring the latter's position and any change thereof. Hence, accurate PM measurements were obtained only for a small number of PNe.

Proper motion measurements have been published for some individual objects. They include spectacular examples of interaction with the ambient interstellar medium (ISM) such as the CS of Sh 2-68 whose PM is aligned with a 45 arcmin long $\mathrm{H} \alpha$ tail formed by matter stripped off the PN (Kerber et al. 2002) or the large filametary PN Sh 2-188 whose arc-shape is also aligned with its PM (Wareing et al. 2006). Combined with distances and radial velocity measurements, proper motions also allow to trace the Galactic orbit of CSs and eventually identify their membership to different stellar populations; Sh 2-216 is a case in point (Kerber et al. 2004; Rauch et al. 2007). See Pauli et al. (2003) for details on the orbital calculations and Galactic stellar populations membership.

On the other hand, no systematic proper motion compilation has been carried out in recent years.

The next major step in the determination of proper motions can be expected from the GAIA satellite (Perryman et al. 2001), 
Table 1. Main properties of the proper motion catalogues used in this work. For each catalogue we list: the $99 \%$ magnitude completeness range $(\Delta m)$, the limiting magnitude $\left(m_{\mathrm{lim}}\right)$, the reference passband, the maximum time baseline $(\Delta T)$ in years, the reference epoch, the absolute average positional $\left(\sigma_{\alpha}, \sigma_{\delta}\right)$ and proper motion accuracies $\left(\sigma \mu_{\alpha}, \sigma \mu_{\delta}\right)$ in right ascension and declination, in mas and mas $\mathrm{yr}^{-1}$, respectively.

\begin{tabular}{l|llllllcll|l}
\hline \hline Catalogue & $\Delta m$ & $m_{\lim }$ & Band & $\Delta T$ & Epoch & $\delta_{\alpha}$ & $\delta_{\delta}$ & $\sigma \mu_{\alpha}$ & $\sigma \mu_{\delta}$ & Reference \\
\hline Hipparcos & $7.3-9$ & 12.4 & $H_{p}$ & $1989.8-1993.2$ & 1991.25 & 0.77 & 0.64 & 0.88 & 0.74 & Perryman et al. (1997) \\
Tycho-2 & $1-11$ & 15.2 & $V_{T}$ & $1989.8-1993.2$ & 2000.00 & 60 & 60 & 2.5 & 2.5 & Høg et al. (2000) \\
UCAC-2 $^{1}$ & $\mathrm{n} / \mathrm{a}$ & 16.0 & $R$ & $1998.1-2002.9$ & 2000.00 & $15-70$ & $15-70$ & $1-7$ & $1-7$ & Zacharias et al. (2004) \\
USNO-B & $1-21$ & 22.0 & $V_{J}$ & $1950-1998$ & 2000.00 & 200 & 200 & $<20$ & $<20$ & Monet et al. (2003) \\
GSC-2 & $1-20.5$ & 22.5 & $F$ & $1950-1998$ & $1987-1998$ & 250 & 250 & $<10$ & $<10$ & in preparation \\
\hline
\end{tabular}

${ }^{1}$ In its present release, the $U C A C-2$ catalogue does not cover the declination strip $+40^{\circ} \leq \delta \leq+52^{\circ}$ and the magnitude completeness is not uniform.

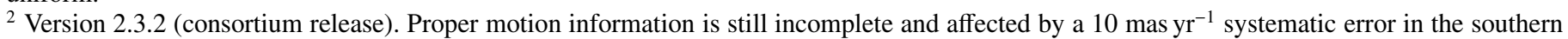
hemisphere $\left(\delta \leq-25^{\circ}\right)$. The positions reported in the GSC-2 catalogue have not been normalized to a given reference epoch but refer to the mean epoch of the most recent observations.

which will provide both highly improved astrometric accuracy and much fainter limiting magnitudes with respect to Hipparcos. Therefore it will deliver PM values for many additional PNe. Currently scheduled for launch in December 2011, final results from GAIA will not become available before 2018 .

Apart from Hipparcos (Perryman et al. 1997) and Tycho-2 (Høg et al. 2000), several proper motion catalogues covering the whole sky (or large areas) have been released in the recent years, e.g. the USNO-B (Monet et al. 2003), the UCAC-2 (Zacharias et al. 2004), the Lick NPM (Hanson et al. 2004), the Yale/San Juan SPM (Platais et al. 1998) the SDSS USNO-B (Gould \& Kollmeier 2004), the PM2000 (Ducourant et al. 2006) and the $G S C$-2 (in preparation). All together, these catalogues provide a large and accurate astrometric database which enables us to embark on a dedicated investigation of proper motion of Galactic PNe.

Here we present a proper motion compilation for a selected subsample of known PNe and CSs using available proper motion catalogues. The structure of the paper is the following: Sect. 2 describes the catalogue matching, Sect. 3 presents the derived proper motion master catalogue, while the comparison with Cudworth's catalogue is discussed in Sect. 4.

\section{The proper motion compilation}

\subsection{The proper motion catalogues}

Since the aim of this work is to carry out a systematic, all-sky, PM survey of PNe, we have focused on existing all-sky PM catalogues while we use other, more region-biased, proper motion catalogues for cross-checking purposes. The astrometric catalogues best suited to our purposes are the Hipparcos final release, the Tycho-2, the UCAC-2, the USNO-B and the GSC-2, which, together, provide all-sky coverage and the best combination between limiting magnitudes and proper motion accuracy. The main properties of these catalogues are summarized in Table 1, more detailed information can be found in the reference publications.

The Hipparcos, the Tycho-2, the UCAC-2, and the GSC-2 catalogues are astrometric reference catalogues and the quality of the astrometric parameters of the first two have been verified several times, e.g. Urban et al. (2000). Moreover the GSC-2 and $U S N O-B$ catalogues are very valuable because of their enhanced magnitude and completeness ranges and also because of the different epochs covered by the original observational data used to compile these catalogues. Hipparcos, Tycho-2, GSC-2, and $U C A C-2$ are all based on the International Celestial Reference System (ICRS).
It is important to note that as pointed out in Monet et al. (2003) the USNO-B proper motions are relative to the YS4.0 catalog and not absolute. This in effect means that the proper motions have systematic errors corresponding to the mean proper motion of stars with $V \sim 17-18$. The size of these systematic errors should be less than the adopted random error minimum of

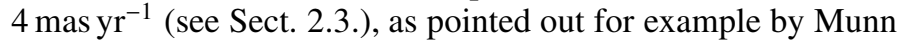
et al. (2004) where they find a systematic error of 3.7 mas/yr with respect to the 3000 square degrees of the SDSS DR1 (Abazajian et al. 2003). As a result the PM values from USNO-B cannot be directly compared with the other results in Table 3.

\subsection{Catalogue matching}

As a starting point for our work, we have used the catalogue of updated PNe coordinates recently published by Kerber et al. (2003a, hereafter Paper I), which are based on the GSC-2. From this catalogue we have extracted a preliminary list which includes both CSs, and PNe which are classified as stellar-like (1123 objects in total). We have then cross-matched the coordinates of the PNe in this list with those of the objects in the selected proper motion catalogues (see previous section) by running dedicated queries either through the Vizier interface ${ }^{1}$ for the public ones, or through restricted access to the consortium object database for the $G S C$-2. The parameters of the queries were fine tuned in order to filter out non useful entries like, e.g., those with non valid proper motion values and/or proper motion errors. In all cases, we have used a matching radius of 1 arcsec which accounts both for the uncertainty on the coordinates of our input list ( 0.25 per coordinate, i.e. the one of the $G S C-2)$ and for the absolute astrometric accuracies of the proper motion catalogues (see Table 1).

After running the cross correlations we found 22 matches with Hipparcos, 56 with Tycho-2, 270 with UCAC-2, 173 with $U S N O-B$, and 993 with the $G S C-2$. The differences in the number of matches are due to the different limiting magnitudes of the catalogues (e.g. between Tycho-2 and UCAC-2), to the different magnitude completeness, and to the different proper motion completeness and sensitivity (e.g. between UCAC-2, USNO-B, and $G S C-2)$. Because of the differences in the positions reference epochs in our input list and in the reference proper motion catalogues (except the $G S C$-2), we have carefully verified that the adopted size of the matching radius (1 arcsec) was large enough to account for the relative positional offsets due to the objects proper motions. We have thus run again all the previous cross-matches using a more generous matching radius

\footnotetext{
${ }^{1}$ http://vizier.u-strasbg.fr/
} 
of 3 arcsec. Although we have indeed found more objects in the Tycho-2, UCAC-2, and USNO-B catalogues with non-zero proper motions, in most cases they are by far too small to explain the positional offsets with respect to the input PNe coordinates. We have found only four objects in the USNO-B catalogue with apparently large enough proper motions, which we have visually inspected. Of these objects, three are associated with extended $\mathrm{PNe}$, and are most likely misclassified emission knots. Only one object is real and associated with a PN with a known CS (NGC 6058). In addition, both its proper motion magnitude $\left(\mu_{\alpha}=132 \pm 50 \mathrm{yr}^{-1} ; \mu_{\delta}=-718 \pm 191 \mathrm{yr}^{-1}\right)$ and direction are consistent with the measured offset with respect to the catalogued CS position. However, according to the GSC-2 this object has a much smaller and not significant proper motion $\mu_{\alpha}=27.3 \pm 18.0 \mathrm{yr}^{-1}$ and $\mu_{\delta}=-27.4 \pm 28.0 \mathrm{yr}^{-1}$. Thus, we consider this as a doubtful case.

As a PN can have entries in more than one catalogue, depending both on its flux and on the magnitude of the PM, the results of the single cross-correlations have then been merged to produce a unique master list as a reference for our proper motion compilation. The merging has been done through a new positional cross correlation between single lists using the same cross-correlation radius as before. To summarize, we found 1031 PNe, i.e. $\sim 90 \%$ of our original input list, which have entries in at least one of the reference catalogues.

\subsection{Filtering and grading}

After creating the master list, we have assessed the proper motion errors across all catalogues. While the nominal proper motion errors of Hipparcos, Tycho-2, and UCAC-2 are reliable, in the case of $U S N O-B$ it has been shown that errors smaller than $4 \mathrm{mas}^{-1}$ are to be considered underestimates (Gould 2003). We have thus reset these errors to a conservative value

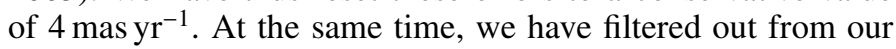
list $U S N O-B$ entries with both right ascension and declination proper motion errors larger than 20 mas $^{-1}{ }^{-1}$, which are considered unreliable (Gould 2003). Then, we have filtered out GSC-2 entries for objects with $\delta \leq-25^{\circ}$, which have been found to be

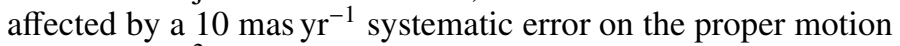
measurement ${ }^{2}$.

The errors and reliability of the $G S C-2$ proper motions with $\delta>-25^{\circ}$ were confirmed using a direct comparison to the $U C A C-2$ catalog.

After these steps, we have recomputed the formal proper motion significance for all the available entries to select only those objects which have a proper motion $\geq 3 \sigma$ in at least one component, and in at least one catalogue. We limit this criterion to one component since some objects have intrinsically low PM in the other component. At the level of accuracy provided by the catalogues many such objects would not qualify if the $\geq 3 \sigma$ filter would be applied to both components removing physically solid PM objects. In this way, we ended up with a clean working sample of 274 objects.

In order to evaluate the consistency across all the available proper motion measurements, we have cross-checked the different entries. In addition, we have used the photometric information given in the catalogues as a secondary cross-checking criteria. Given the published accuracies and the overlapping photometric bands this approach should be a safe measure for most, if not all, of the objects in our list. Of course, we have applied this criteria with due caution in order not to accidently discard

${ }^{2}$ http://www-gsss.stsci.edu/Catalogs/GSC/GSC2/GSC2.htm

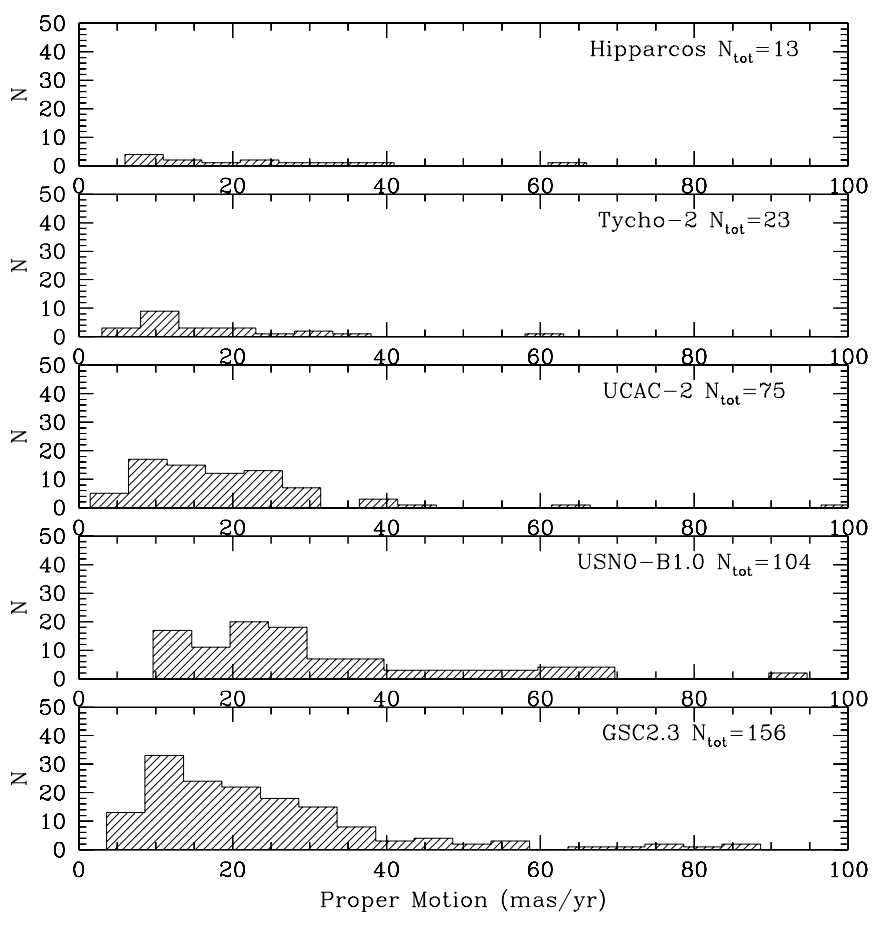

Fig. 1. Histograms of the proper motion modulus of all objects in Table 3 fulfilling our $3 \sigma$ filtering. The numbers on the top right give the total number of objects in each catalogue. USNO-B has been included for comparison, although it is not used in our proper motion grading.

variable objects and/or objects with extreme colors. For each object we have then defined a confidence classification scheme which takes into account: (i) the proper motion significance in each catalogue, (ii) the agreement between the different available proper motion measurements, and (iii) the number of compatible proper motion measurements across all catalogues. According to these guidelines we have defined four gradings as follows:

- $A$ : proper motion at $\geq 5 \sigma$ in at least one component, confirmed by at least one other independent catalogue $(\geq 3 \sigma)$;

- $B$ : proper motion at $3 \leq \sigma \leq 5$ in at least one component, confirmed by at least one other independent catalogue;

- $C$ : proper motion at $\geq 3 \sigma$ in at least one component, in one catalogue only;

- $D$ : proper motion at $\geq 3 \sigma$ in at least one component, but discrepancies with other catalogues or a large error bar ( $\geq 30$ mas) make the significance questionable,

and for each object we have assigned a proper motion grading accordingly. As discussed in Sect. 2.1. the PM values from $U S N O-B$ are not absolute but relative to YS4.0. Hence these data have not been included when doing the above grading. It is quite reassuring to see that the number of objects with (significant) discrepant results from different catalogues is very low.

\section{The proper motion master catalogue}

The full final proper motion master catalogue includes 274 objects and is listed in Table 3 that is only available in electronic form at the CDS. Figure 1 shows the histograms of the proper motion modulus from the values listed in Table 3. Only proper motions fulfilling our $3 \sigma$ filtering have been included in the histograms. The lower contribution of the Hipparcos and Tycho-2 catalogues has to be ascribed to the faintness of our targets which are mostly fainter than $V \sim 15$. As seen, the contribution of the 
$G S C$-2 has been fundamental to include proper motions for the faintest objects of our starting sample and thus to significantly enlarge our compilation.

According to the classification defined above, we obtained 20 objects with proper motions in category A, 7 in B, 207 in C, and 40 in D. All objects in category D formally fulfill the $3 \sigma$ criterion but they have to be considered doubtful cases because of discrepant results from other catalogues or high absolute PM

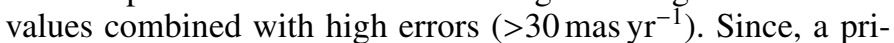
ori, we have no compelling reason to give more weight to one catalogue with respect to another, we did not make an attempt to resolve the discrepancies but flagged all such objects as doubtful. The origin of the, at least partially, discrepant proper motion values will not be explored in this work but it is likely to be related to some random error in the catalogues rather than to an object mismatch.

Thus, we ended up with a robust proper motion compilation for 234 objects, (plus another 40 doubtful cases) i.e. $\sim 25 \%$ of the original sample.

We have also checked how the morphological classification affects the proper motion measurements of the sample. One would expect that proper motions are more easily and reliably measurable for stellar-like objects or for CSs. Indeed, such a correlation clearly emerges from the analysis of our sample. For this analysis we have used the morphological classifications given in Paper I: CS, in the case of presence of a well-defined central star. ST, for a stellar-like, and PHOT for an extended object. We found that for objects with PM 71 belong to the CS class, 80 to the ST class and 123 to the PHOT class. Thus, it seems that the object morphology does not represent an immediate discriminant for the proper motion measurement. However, taking the PM grading into account we find that $\sim 65 \%$ of the objects with A-graded proper motions belong to the CS class. For the objects with B-graded proper motions, $\sim 60 \%$ belong either to the CS or to the ST classes while this ratio decreases to $\sim 55 \%$ for objects with $\mathrm{C}$-graded proper motions.

We note that only a few objects have entries in all five catalogues in Table 3, as one would expect from a compilation which is the merging of different catalogues, with different limiting magnitudes, sky coverage, completeness, proper motion detection thresholds. For example, for 89 objects we only have the proper motion entries from the GSC-2. This obviously reflects the different limiting magnitudes of our reference catalogues (see Table 1). The fact that for these objects we have no proper motion entry in USNO-B, which has a limiting magnitude comparable to the $G S C$-2 one, is probably to be ascribed either to the different proper motion completeness of this catalogue and/or to the different proper motion error estimates which determined our $3 \sigma$ threshold cut. Indeed, in general only $30 \%$ of USNO-B objects have non zero proper motions, so the poor success rate for that catalog is not unexpected.

Similarly for 58 objects we have a $U S N O-B$ but not a $G S C$ 2 entry which is explained by the fact that they are mostly in the South, where we have discarded all $G S C$-2 entries due to the reported proper motion systematics. The fact that for $26 \mathrm{ob}-$ jects we have entries only in one (or more) of the bright catalogues, i.e. Hipparcos, Tycho-2 and UCAC-2 and not in the faint ones, i.e. $U S N O-B$ and $G S C-2$ is not surprising as it is mostly related to the magnitude of the proper motions which require smaller errors, unachievable by the former catalogues. We note how the contribution of $U C A C-2$ has been relevant in increasing our proper motion compilation as it covers the intermediate region of objects too faint to be in either Hipparcos or Tycho2 and with proper motions too tiny to be measured by either
$U S N O-B$ or $G S C-2$. Indeed, 25 objects (about $9 \%$ of the total) have only a $U C A C-2$ entry. In addition, $U C A C-2$ has been built on CCD observations, which have a much sharper spatial resolution with respect to the photographic plates used to build both the $U S N O-B$ and the GSC-2. This means that, for instance, objects detected as blends in either of the two catalogues have been clearly resolved in $U C A C-2$, if bright enough.

In this context it is important to keep in mind that orbital motion of a binary central star can in principle lead to a false PM detection. Certain combinations of separation and orbital period could cause a shift in the photo-center of the central star mimicking a PM of its position. While such a combination probably is rare, we know that a significant fraction of PNe have binary central objects. A survey with the HST (Ciardullo et al. 1999) aimed at resolved companions of PN CSs resulted in a fraction of about $10-15 \%$ in a sample of about 100 PNe. Among their probable and possible binaries are a few objects for which subarcsecond companions have been found: A 31, K 1-14, K 1-22, and NGC 6210. These could be relevant in the context of PM derived from the all-sky surveys but the differences in magnitude and colour suggest no significant influence maybe with the exception of K 1-14 and K 1-22. Still, the actual fraction of binary central stars is not well constrained and more recent observations (De Marco et al. 2004) and theoretical work (Maxwell \& De Marco 2006) suggest that binarity could be very common. In fact high quality proper motion data will be a very useful tool to confirm physical association of resolved pairs via their common PM.

\section{Comparison with the Cudworth's proper motion catalogue}

We have compared our proper motion master catalogue with the one of Cudworth (1974) which reports proper motion measurements for $62 \mathrm{PNe}, 51$ originally measured by him and 11 taken from the literature (see Cudworth 1974, and references therein).

Cudworth used a special technique with a rotating sector to reduce the intensity of the mostly bright CSs of his objects. He also had a well defined observing strategy minimizing systematic errors. Cudworth used a refractor with a long f-ratio and reported very small PM values with errors as small as $1{\text { mas } \mathrm{yr}^{-1}}^{-1}$ for a time base of about one decade. Our data is based on general purpose surveys. For the bulk of the data the errors on the PM are of order a few mas $\mathrm{yr}^{-1}$. In this sense Cudworth's dedicated PM work was not only ahead of its time but is still hard to match today.

We have made the comparison with our master catalogues for all of the 62 Cudworth objects and found corresponding entries for 41 of the objects. It is a bit surprising that only $2 / 3$ of Cudworth objects are recovered by this compilation based on several all-sky catalogues. We find several reasons for this.

The majority of Cudworth's objects are NGC objects with bright PN nebulosity which can significantly interfere with accurate measurements of the PM of their CSs. Three objects have been classified as extended PNe in Paper I and hence have been excluded by our pre-selection (see Sect. 2.2). Also, our survey catalogues are not complete in terms of PM data. The present release of the UCAC-2 catalogue, does not cover the full sky and the magnitude completeness is not uniform. For USNO-B and $G S C$-2 the proper motion information is still incomplete.

For the comparison we have processed Cudworth's results from FK4 to the FK5 framework. The remaining differences to the ICRS used by the modern catalogues are fractional mas $\mathrm{yr}^{-1}$ 
Table 2. Comparison with Cudworth (1974) and other proper motion results from the literature. The table only contains Cudworth objects for which a meaningful comparison with our results is possible. Results from Cudworth have been processed from FK4 to FK5. The first part of the table contains objects that had a significant $(\geq 3 \sigma)$ proper motion modulus in Cudworth's catalogue, the second part of the table contains objects that had proper motion modulus $<3 \sigma$ in Cudworth's for which we found significant PM, the bottom part lists objects for which we found other PM data in the literature. Objects for which our results provide in an improvement in accuracy of the PM modulus with respect to the results by Cudworth or other references are typeset in bold. The comparison is also illustrated in Fig. 2.

\begin{tabular}{l|cc|ccc|cc}
\hline \hline & \multicolumn{2}{|c|}{ Cudworth in FK5 } & \multicolumn{3}{c|}{ this compilation } & \multicolumn{2}{c}{ literature } \\
PN name & $\mu_{\alpha}$ & $\mu_{\delta}$ & $\mu_{\alpha}$ & $\mu_{\delta}$ & Source & $\mu_{\alpha}$ & $\mu_{\delta}$ \\
\hline NGC 246 & $-17.9 \pm 1.0$ & $-15.4 \pm 4.2$ & $-22.0 \pm 1.5$ & $-8.3 \pm 1.2$ & $U C A C-2$ & - & - \\
A 33 & $-14.8 \pm 3.9$ & $10.0 \pm 2.4$ & $-25.3 \pm 4.0$ & $1.7 \pm 8.0$ & $G S C-2$ & - & - \\
A 36 & $20.5 \pm 3.7$ & $1.0 \pm 3.6$ & $12.0 \pm 1.4$ & $3.5 \pm 1.3$ & $U C A C-2$ & - & - \\
NGC 6309 & $8.5 \pm 3.7$ & $14.1 \pm 4.1$ & $-25.6 \pm 28.0$ & $63.7 \pm 24.0$ & $G S C-2$ & - & - \\
NGC 6629 & $-1.2 \pm 1.2$ & $6.5 \pm 1.0$ & $-2.8 \pm 1.8$ & $1.3 \pm 1.8$ & $U C A C-2$ & - & - \\
NGC 6751 & $1.4 \pm 1.2$ & $-5.4 \pm 1.0$ & $-9.2 \pm 3.0$ & $-5.9 \pm 3.1$ & $U C A C-2$ & - & - \\
M 1-67 & $-0.4 \pm 1.0$ & $-10.6 \pm 2.5$ & $-3.1 \pm 1.0$ & $-6.2 \pm 1.4$ & $U C A C-2$ & - & - \\
BD+303639 & $-0.5 \pm 1.2$ & $-22.4 \pm 4.0$ & $-3.0 \pm 0.6$ & $-8.7 \pm 0.6$ & $U C A C-2$ & - & - \\
NGC 6833 & $15.6 \pm 2.3$ & $-1.0 \pm 3.3$ & $-7.6 \pm 2.0$ & $-5.7 \pm 2.0$ & $G S C-2$ & - & - \\
NGC 6891 & $2.1 \pm 1.0$ & $-9.6 \pm 3.1$ & $3.8 \pm 1.5$ & $-3.4 \pm 3.2$ & $U C A C-2$ & - & - \\
IC 5117 & $21.4 \pm 3.0$ & $-4.8 \pm 4.3$ & $13.9 \pm 16.0$ & $-3.0 \pm 10.0$ & $G S C-2$ & - & - \\
NGC 7293 & $42.0 \pm 2.8$ & $-5.0 \pm 1.9$ & $37.3 \pm 2.5$ & $-3.9 \pm 1.8$ & $U C A C-2$ & $30 \pm 5$ & $-1 \pm 0^{2}$ \\
\hline NGC 1514 & $-2.9 \pm 1.7$ & $-4.3 \pm 3.6$ & $-5.9 \pm 0.6$ & $2.7 \pm 0.8$ & $U C A C-2$ & - & - \\
IC 4593 & $-4.7 \pm 2.1$ & $1.1 \pm 3.4$ & $-6.5 \pm 1.2$ & $2.8 \pm 1.2$ & $U C A C-2$ & $-11.4 \pm 1.7$ & $3.1 \pm 1.6^{1}$ \\
NGC 6210 & $-0.4 \pm 2.5$ & $-6.9 \pm 7.5$ & $1.3 \pm 1.0$ & $-13.2 \pm 0.8$ & $U C A C-2$ & $-9.6 \pm 3.8$ & $-16.0 \pm 3.8^{3}$ \\
NGC 6644 & $-15.5 \pm 5.4$ & $-7.9 \pm 6.1$ & $-12.9 \pm 1.7$ & $-8.8 \pm 1.6$ & $U C A C-2$ & - & - \\
IC 4732 & $4.0 \pm 1.0$ & $3.7 \pm 4.2$ & $-14.3 \pm 4.0$ & $17.4 \pm 4.0$ & $G S C-2$ & - & - \\
\hline EGB 4 & - & - & $-3.1 \pm 4.0$ & $-33.8 \pm 4.0$ & $G S C-2$ & $3.9 \pm 4.0$ & $-25.1 \pm 1.8^{4}$ \\
He 2-131 & - & - & $-3.0 \pm 1.0$ & $-2.6 \pm 1.6$ & $U C A C-2$ & $-7.9 \pm 2.7$ & $-2.0 \pm 2.4^{3}$ \\
NGC 3132 & - & - & $-8.1 \pm 1.0$ & $1.2 \pm 1.2$ & $U C A C-2$ & $-9.0 \pm 2.4$ & $5.7 \pm 2.1^{1}$ \\
NGC 7094 & - & - & $4.4 \pm 1.5$ & $-10.0 \pm 1.5$ & $U C A C-2$ & $3.5 \pm 0.8$ & $-10.9 \pm 0.8^{5}$ \\
Sh 2-68 & - & - & $-22.6 \pm 4.0$ & $-52.8 \pm 4.0$ & $G S C-2$ & $-19.9 \pm 5.6$ & $-49.3 \pm 5.5^{6}$ \\
\hline
\end{tabular}

${ }^{1}$ Høg et al. (1998), ${ }^{2}$ Harrington et al. (1980), ${ }^{3}$ Röser \& Bastian (1988), ${ }^{4}$ Greiner et al. (2001), ${ }^{5}$ Ducourant et al. (2006), ${ }^{6}$ Kerber et al. (2002).

and are not relevant given the overall accuracy. We have used the proper motion modulus as a metric for the comparison provided the PM vector points in the same direction.

In addition it is important to keep in mind that not all the proper motion measurements reported by Cudworth are significant. Applying a $3 \sigma$ filter on the 41 matches we find that only 12 of Cudworth's objects have a proper motion modulus (in FK5) larger than $3 \sigma$. For these 12 objects a meaningful comparison can be made and the results are given in Table 2 and Fig. 2. The first two columns give the PM values as reported by Cudworth (1974) but processed to FK5, the next three give the best PM found by us and its source, and the final two provide any additional PM information available in the literature. The upper section of the table contains the objects with significant $(\geq 3 \sigma)$ PM modulus in Cudworth's catalogue, the second section lists the five objects with $\sigma<3$ for which a significant result has been found by us, and the bottom section contains objects not reported by Cudworth but elsewehere in the literature. The 9 objects for which the new values improve the accuracy of Cudworth's $\mathrm{PM}$ results are set in bold. This is the case for five (A 36, BD+30 3639, M 1-67, NGC 246, NGC 7293) of Cudworth's 12 significant objects for which our results are also significant at the $3 \sigma$ level. NGC 6833 is a special case in that agreement is very good for the PM modulus but in fact the signs of the PMs in RA are opposite. It is very reassuring that there is only one such case where significant results of Cudworth and our work are in disagreement.
In summary, using the proper motion modulus as a metric and a $\sigma \geq 3$ filter there are 12 Cudworth objects and good agreement is found for 10 of these (Fig. 2). For five cases in which both Cudworth and our results are significant we are able to improve the accuracy of the PM (bold in upper section of Table 2), while NGC 6833 is discrepant. In addition we found significant PM for five (middle section of Table 2) of Cudworth non-significant objects. Excellent agreement is also found with the few examples of PM data from other publications (Fig. 2 bottom panel).

\section{Conclusions and outlook}

We have performed a compilation of the proper motions for a sample of PNe and CSs using as reference all available all-sky astrometric catalogues. Starting from a sample of 1123 objects selected from the catalogue presented in Paper I we have derived proper motion information for a total of $234 \mathrm{PNe}$ and CSs plus another 40 doubtful candidates, all formally significant at the $\geq 3 \sigma$ level in at least one component, albeit with different degrees of confidence, as expressed by our classification. Compared to the original work of Cudworth (1974), who listed 62 (25 significant in at least one component) objects in total, our compilation thus provides a factor of more than 3 increase in the number of $\mathrm{PNe}$ with available proper motion information but a factor of more than 9 increase for objects with $\mathrm{PM} \geq 3 \sigma$ in at least one component. Our proper motion compilations contains a total of about 190 objects with new proper motions measurements $(>3 \sigma$ 

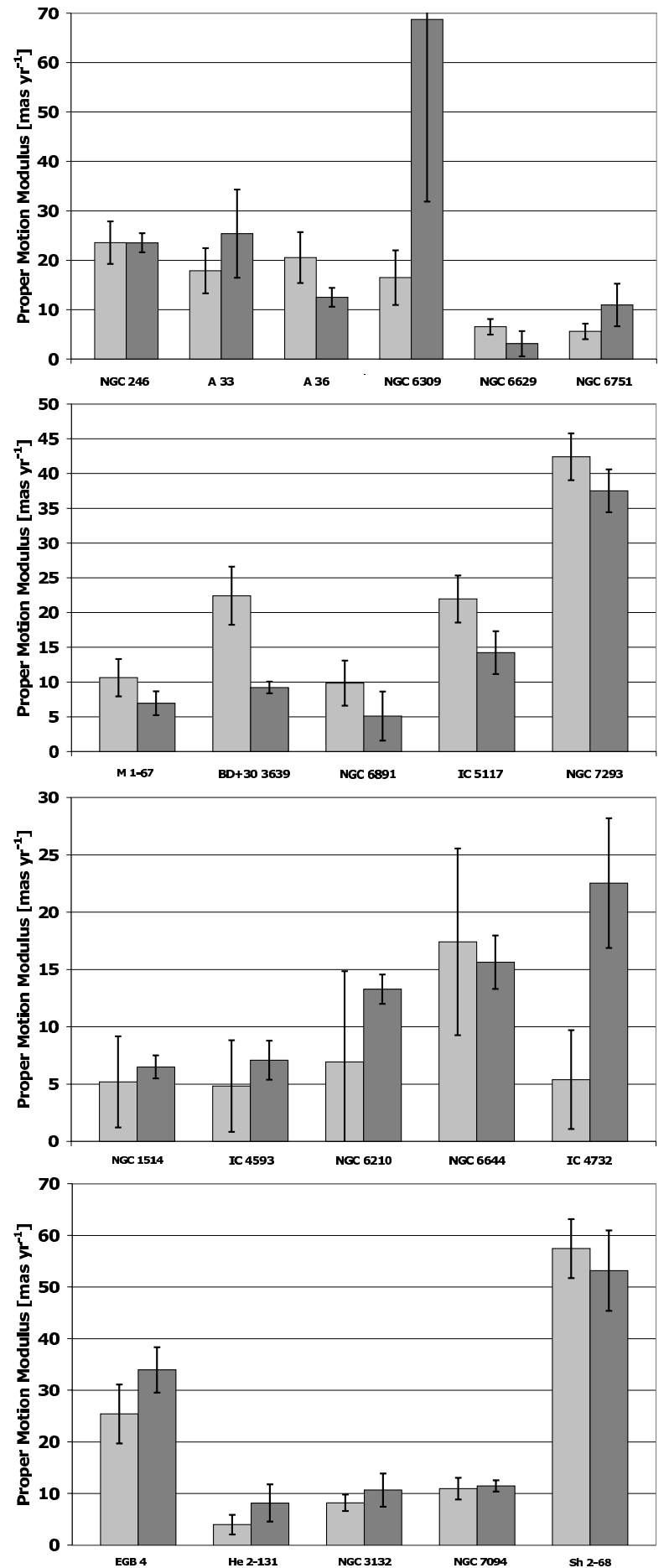

Fig. 2. Proper Motion Modulus for objects in Table 2. Cudworth's values (in FK5 reference frame) are in light grey, while our compiled results are in dark grey. The top two panels gives the objects with a significant $(\geq 3 \sigma)$ proper motion modulus in Cudworth's catalogue; the third panel contains objects with significance $<3 \sigma$ in Cudworth's catalogue but $>3 \sigma$ found by us. The bottom panel shows objects for which we found other PM data in the literature.

in at least one component). The total number of PNe with known PM was about 40 (25 from Cudworth (1974), 11 from Hipparcos (Acker et al. 1998) and a few others form the literature (five objects are given in Table 2 but the census of the literature may be incomplete). Hence our work represents an increase in the total number of PNe with PM of about 5.
This compilation can now serve as a reference for future work. PM information will be be highly valuable in the further study of the interaction between the PNe and their ambient ISM e.g. Kerber et al. (2003b). We will use this PM sample to conduct a study of the galactocentric orbits of PNe and CSs and study their membership to the various Galactic stellar populations (Kerber et al. 2004). Such work is also relevant for extragalactic PNe. The PN luminosity function which is used for distance determination is dependent on the PN population in that galaxy (Marigo et al. 2004; Sambhus et al. 2005). It would also be very interesting to obtain PM from space-based observations of extragalactic PNe. A possible detection of PM would add valuable information to studies of galaxy dynamics and the presence of dark matter.

Acknowledgements. We wish to thank an anonymous referee for his valuable comments that helped us improve the paper. This research has made use of the infrastructure provided by the EURO-VO project. We have made use of the SIMBAD database and in particular the VizieR Service, operated at CDS, Strasbourg, France. We thank the $G S C$-2 consortium for granting access to data prior to publication. The Guide Star Catalogue-II is a joint project of the Space Telescope Science Institute and the Osservatorio Astronomico di Torino. The Space Telescope Science Institute is operated by the Association of Universities for Research in Astronomy, for the National Aeronautics and Space Administration under contract NAS5-26555. The participation of the Osservatorio Astronomico di Torino is supported by the Italian Council for Research in Astronomy. Additional support is provided by European Southern Observatory, Space Telescope European Coordinating Facility, the International GEMINI project and the European Space Agency Astrophysics Division.

\section{References}

Abazajian, K., Adelman-McCarthy, J. K., Agüeros, M. A., et al. 2003, AJ, 126, 2081

Acker A., Fresneau, A., Pottasch, S. R., \& Jasniewicz, G. 1998, A\&A 337, 253

Ciardullo, R., Bond, H. E., Sipior, M. S., et al. 1999, AJ, 118, 488

Cudworth, K. 1974, AJ, 79, 1384

Harrington, R. S., \& Dahn, C. C. 1980, AJ, 85, 454

De Marco, O., Bond, H. E., Harmer, D., \& Fleming, A. J. 2004, ApJ, 602, L 93 Ducourant, C., Le Campion, J. F., Rapaport, M., et al. 2006, A\&A, 448, 1235

Gould, A., 2003, AJ, 126, 472

Gould, A. R., \& Kollmeier, J. A. 2004, ApJS, 152, 103

Greiner, J., Tovmassian, G., Orio, M., et al. 2001, A\&A, 376, 1031

Hanson, R. B., Klemola, A. R., Jones, B. F., \& Monet, D. G. 2004, AJ, 128, 1430 Harris, H. C., Dahn, C. C., Canzian, B., et al. 2007, AJ, 133, 631

Høg, E., Kuzmin, A., Bastian, U., et al. 1998, A\&A, 335, L65

Høg, E., Fabricius, C., Makarov, V. V., et al. 2000, A\&A, 355, L27

Kerber, F., Guglielmetti, F., Mignani, R. P., \& Roth, M. 2002, A\&A 381, L 9

Kerber, F., Mignani, R. P., Guglielmetti, F., \& Wicenec, A. 2003a, A\&A 408, 1029 (Paper I)

Kerber, F., Guglielmetti, F., Mignani, R. P., \& Roth, M. 2003b, in Sun Kwok, ed. M. Dopita, \& R. Sutherland, Planetary Nebulae: Their Evolution and Role in the Universe (San Francisco: ASP), Proc. IAU Symp., 209, 525

Kerber, F., Mignani, R. P., Pauli, E.-M., Wicenec, A., \& Guglielmetti, F. 2004, A\&A 420, 207

Marigo, P., Girardi, L., Weiss, A., Groenewegen, M. A. T., \& Chiosi, C. 2004, A\&A, 423, 995

Maxwell, M., \& De Marco, O. 2006, ApJ, 650, 916

Monet, D. G., Levine, S. E., Canzian, B., et al. 2003, AJ, 125, 984

Munn, J. A., Monet, D. G., Levine, S. E., et al. 2004, AJ, 127, 3034

Pauli, E.-M., Napiwotzki, R., Altmann, M., et al. 2003, A\&A, 400, 877

Perryman, M. A. C. 2001, Ap\&SS, 280, 1

Perryman, M. A. C., Lindegren, L., Kovalevsky, J., et al. 1997, A\&A, 323, L49

Platais, I., Girard, T. M., Kozhurina-Platais, V., et al. 1998, AJ, 116, 2556

Rauch, T., Ziegler, M., Werner, K., et al. 2007, A\&A, 470, 317

Röser, S., \& Bastian, U. 1988, A\&AS, 74, 449

Sambhus, N., Gerhard, O., \& Méndez, R. H., 2005, in Planetary Nebulae as Astronomical Tools, AIP Conf. Proc., 804, 317

Urban, S. E., Wycoff, G. L., \& Makarov, V. V. 2000, AJ, 120, 501

Wareing, C. J., O'Brien, T. J., Zijlstra, A. A., et al. 2006, MNRAS, 366, 387

Zacharias, N., Urban, S. E., Zacharias, M. I., et al. 2004, AJ, 127, 3043 